\title{
Efficacy of Including Interferential Current Therapy in the Rehabilitation Program of Children with Functional Constipation
}

\author{
IKRAM I. ALYAN, M.D.* and MONA S.H. MAKHLOUF, M.D. \\ The Departments of Rheumatology \& Rehabilitation* and Pediatrics**, El-Galaa Teaching Hospital, Cairo
}

\begin{abstract}
Aim: To determine the effectiveness of addition of interferential current (IF) stimulatory current to the other conventional therapeutic tools related to childhood functional constipation (FC).

Methods: Children suffering from FC were recognized according to Rome-III criteria of constipation. Two treatment groups, the case group $(n=40)$ underwent IF electrical stimulation added to pelvic floor muscle (PFM) exercises and the control group $(n=40)$ received PFM exercises without IF stimulation. A full bowel history with regarding data on defecation frequency/week, form of stool, and the number of fecal soiling episodes/day), a constipation score questionnaire, a visual pain score, a constipation related quality of life questionnaire (QOL) were noted before, after the treatment sessions and 6 months later for all participants.
\end{abstract}

Results: The median of constipation score was decreased in both groups with the cases having lower scores after the treatment ( $p<0.089$ and $p<0.0001$ respectively). Significantly a better outcome for pain score $(p<0.0001)$ was also observed among the cases after the 6 months follow-up. Fecal soiling episodes/day were reduced after treatment in both groups; this finding was significant in the case group in relation to controls. The constipation-related QOL score has significantly improved all participants.

Conclusion: IF stimulatory current was associated with better results in the efficacy of treatment of chronic cases suffering from FC and it should be added to conventional therapy as a part of the rehabilitation program.

Key Words: Interferential current - Functional constipation - Children.

\section{Introduction}

FUNCTIONAL constipation (FC) represents a common health problem affecting children. The prevalence is about $15 \%$ [1]. The main manifestations of FC were infrequent bowel movements,

Correspondence to: Dr. Ikram I. Alyan, The Department of Rheumatology \& Rehabilitation, El-Galaa Teaching Hospital, Cairo hard and/or large stools, and painful defecation and associated abdominal pain [2], which can affect children well-being and health-related quality of life [3]. The first-line therapy, based on medical treatment combined with laxatives and dietary rules, behavioral modifications, pelvic floor (PFM) exercises and toilet training is often disappointing. Children with resistant constipation remains a difficult problem, and face surgical procedures [4] Promising results have been achieved with innovative therapies such as transcutaneous neuromodulation through Interferential (IF) current electrical stimulation [5] for the treatment of such cases. Two sinusoidal stimulatory torques crossing the body are thought to stimulate peripheral nerves [6]. This stimulation may lead to modulation of extrinsic neural control of the large bowel or modulation of reflexes that inhibit large bowel function [6]. Our purpose was to study the efficacy of adding IF current therapy to other conventional therapeutic tools related to childhood functional constipation.

\section{Patients and Methods}

One hundred children (5-15 years) referred from pediatric clinic with persistent constipation were evaluated. 20 children were excluded because of lacking of inclusion criteria of FC. The study was conducted in rehabilitation clinic from January 2016 to May 2017. The study protocol was reviewed and approved by the ethics committees of El-Galaa teaching hospital. Parents gave written informed consent. 80 children were recorded and followed the treatment protocol for 6 months and they divided randomly into two equal groups, either the IF effective stimulation, case group or sham stimulation, the control group. A complete physical examination was done for all participants with special attention to back and spine. Also neurolog- 
ical, rectal and anal sphincter examinations were done.

\section{Inclusion criteria:}

Patients who had functional constipation for at least 6 months were evaluated by a pediatrician and previous interventions were done such as dietary modifications and/or laxatives with failure to see the desirable response. The diagnosis of functional constipation had been confirmed for them based on Rome III criteria [7] as having at least two out of six of the following symptoms for two or more months: Two or fewer defecations per week; at least one episode of fecal incontinence per week; history of retentive posturing or excessive volitional stool retention; history of painful or hard bowel movements; presence of a large fecal mass in the rectum; history of wide diameter stools that may obstruct the toilet.

\section{Exclusion criteria:}

Patients with inflammatory or metabolic diseases. Children with neurologic or psychiatric disease and Hirschsprung's disease, or who had previous history of abdominal or anal sphincter surgeries were also excluded. A pediatric incontinence/constipation score questionnaire [scale 0 to 29] [8] and a visual pain score (scale of 0 to 10,10 being the worst) were recorded. The laxative regimen was continued for all children during the treatment. Patients in both groups were evaluated with a constipation-related QOL questionnaire [9] before and after the end of treatment sessions, and six months after end of the treatment. The QOL questionnaire included 37 questions: 3 items related to constipation, 13 items related to emotional functioning, 11 items related to social functioning and 10 items related to treatment/intervention. Both groups were advised to follow the conventional treatment of functional constipation which consists of simple education about gastrointestinal tract function, use of high fiber diet and fruit, hydration. Correct stool regulation was clarified, patients were asked to sit on the toilet three times a day after mealtime in a relaxed position for $5 \mathrm{~min}$. Toilet training including correct defecation posture and using foot support in short patients was also advised. We described PFM to the parents using colored figures. Both groups were instructed to do PFM exercises. All patients were educated to do PFM contractions for 10 s followed by 30 s of relaxation, abdominal strain, and bear-down maneuver. All patients were asked to do daily home exercises of 10 repetitions under parental supervision. In addition the case group received effective IF electric stimulation; the control group underwent sham stimulation. In accordance with previous study [10] IF current therapy was applied using two electrodes the lower gastrointestinal tract via placement them on the abdomen lateral to the umbilicus and 2 on the back at the level of T9-L2, leads were connected from the right front to the left back and vice versa so that the currents crossed. In the case group, the intensity was increased until the patient declared a strong but comfortable level of sensory alertness without muscle contractions. Patients in the control group also underwent the same procedure without increasing the intensity (Fig. 2). Therapy was administered for 10 courses, $20 \mathrm{~min}$. per session twice per week for all participants using an electrotherapy device (Gymna Company (Belgium) (Fig. 3), the device delivered a $4 \mathrm{kHz}$ carrier frequency for the two currents.

\section{Statistical analysis:}

Statistical analysis was performed using Statistical Package of Social Science software (version 19). Categorical data were reported as frequencies and percentages. To analyze data, $\mathbf{x}_{2}$ or Fisher's exact tests was applied for nonparametric statistical comparisons before and after the treatment in both cohorts. Mann-Whitney U-test and Student's $t$-test were performed wherever applicable, to compare the values between the case and control groups. Data are expressed as median and mean \pm S.D. $p$ levels of $<0.05$ were considered statistically significant. All authors had access to the study data and reviewed and approved the final manuscript.

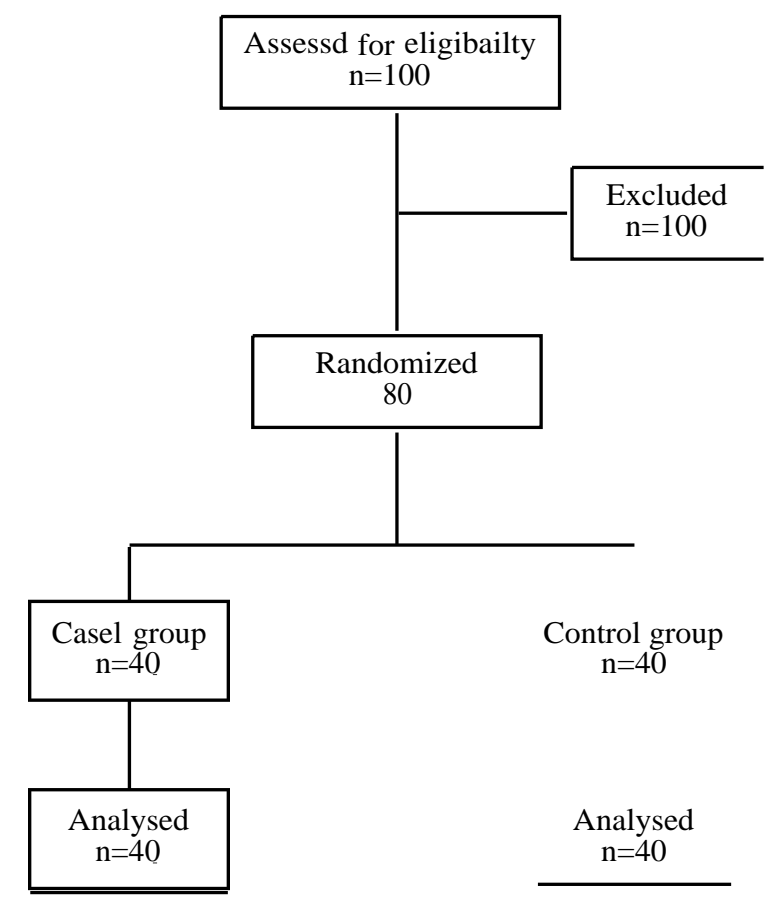

Fig. (1): Flow Diagram of the study. 

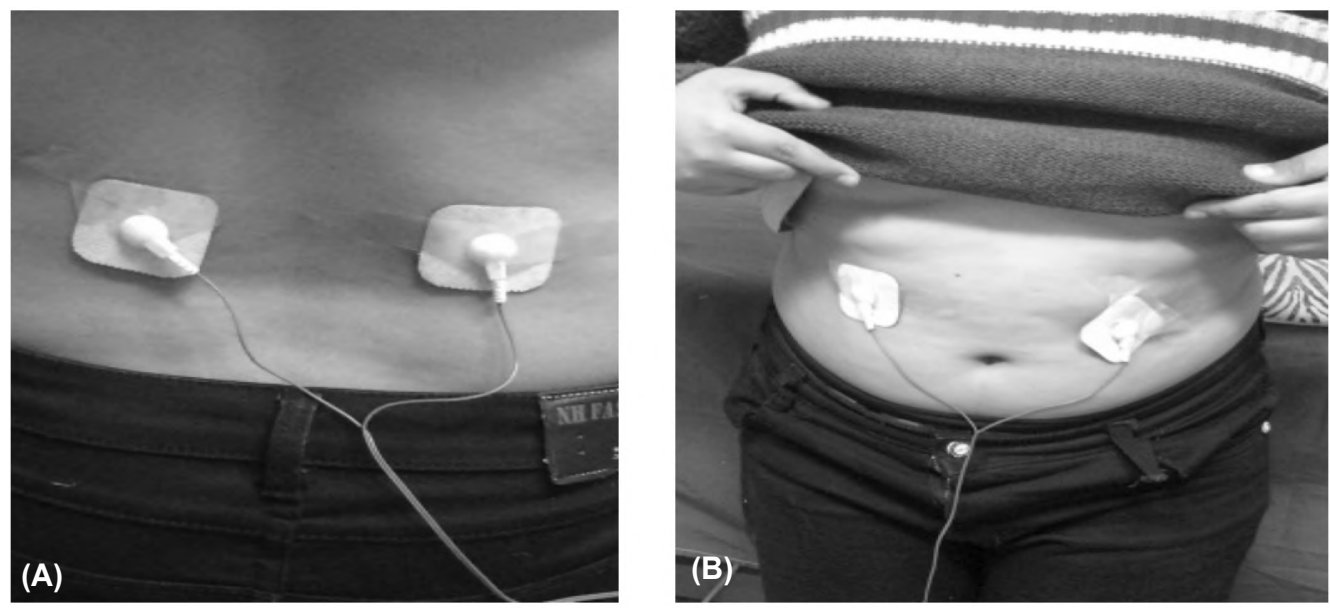

Fig. (2): Position of electrodes on the abdomen (A) and back (B).

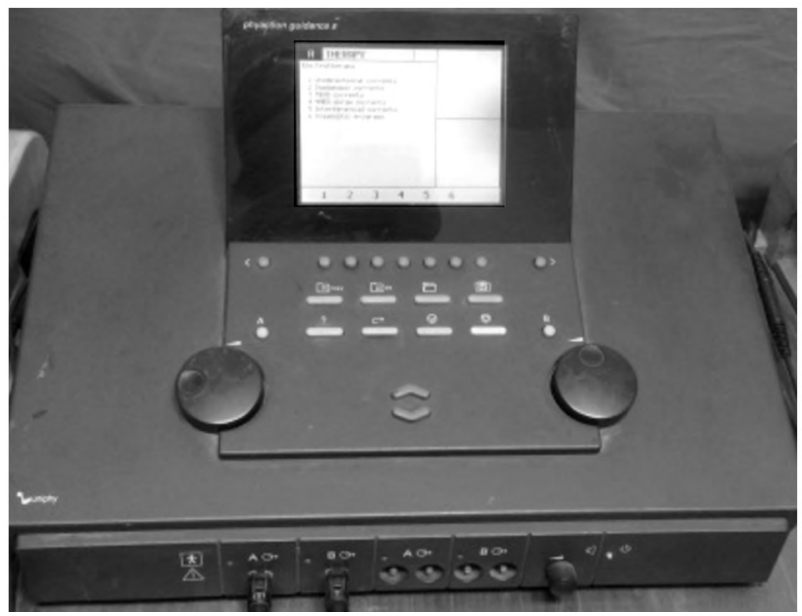

Fig. (3): Electrotherapy device generating interferential current.

\section{Results}

One hundred children suffering from persistent constipation were examined; Fig. (1) showed the flow diagram of participants. No adverse effects throughout treatment sessions or in follow-up period were recorded, also they completed the study, with no dropped out from the analysis. Data in Table (1) clarify the changes between controls and cases before and after the treatment sessions and after 6 months follow-up period. The median of constipation score was reduced in both groups with the cases having significantly lower scores after the treatment $(p<0.089$ and $p<0.0001$ respectively) (Table 1). Significantly better outcomes for pain score $p<0.0001$ ) were also observed among the cases after the 6 months follow-up (Table 1). Fecal soiling episodes/day were reduced after treatment in both groups; this finding was signifi-

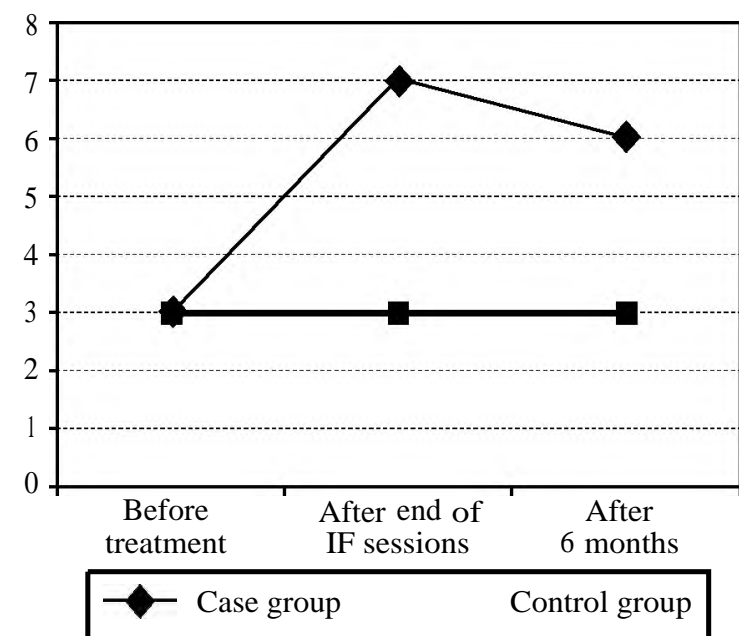

Fig. (4): Median of defecation frequency per week pre and post therapy.

cant in the case group compared with the controls. This difference remained significant $(p<0.0001)$ at 6 months follow-up. The treatment in both groups yielded significant improvement regarding the constipation-related QOL score; however, no differences were observed between the groups after the treatment or after the 6 months follow-up. Table (2) presents changes in all Rome III criteria after the treatment in both groups, which was reported by a pediatrician. The dose of laxative was reduced in patients who responded to the treatment at the end of the treatment sessions; it was discontinued in patients who sustained their response to the treatment after 6 months follow-up.

Table (2) presents changes in all Rome criteria after the treatment in both groups, which was reported by a pediatrician. 
Table (1): Change in constipation symptoms after end of treatment courses and 6 months of follow-up in both groups.

\begin{tabular}{llcll}
\hline Variable (median) & Groups & $\begin{array}{c}\text { Before } \\
\text { treatment }\end{array}$ & $\begin{array}{c}\text { After end of } \\
\text { IF sessions }\end{array}$ & $\begin{array}{c}\text { After } \\
6 \text { months }\end{array}$ \\
\hline Defecation frequency/week & Case group & $3(1)$ & $7(3)$ & $6(3)$ \\
& Control group & $3(1)$ & $3(1)$ & $3(1)$ \\
& $p$-value & 0.997 & $0.01^{*}$ & $0.007 * *$ \\
Fecal soiling episodes/day & Case group & $4(1)$ & $0(0)$ & $0(0)$ \\
& Control group & $3(1)$ & $1(1)$ & $1(1)$ \\
& $p$-value & 0.299 & $0.001 * *$ & $0.001 * *$ \\
Constipation score $(0-29)$ & Case group & $12(5)$ & $5(1)$ & $5(1)$ \\
& Control group & $11(4)$ & $9(3)$ & $9(3)$ \\
Pain score (0-10) & $p$-value & 0.089 & $0.0001 * * *$ & $0.0001 * * *$ \\
& Case group & $5(2)$ & $0(0)$ & $0(0)$ \\
Quality of life score & Control group & $4(2)$ & $0(0)$ & $2(1)$ \\
& $p$-value & 0.087 & 1 & $0.0001 * * *$ \\
& Case group & $58(5)$ & $65(4)$ & $65(6)$ \\
& Control group & $60(6)$ & $60(5)$ & $62(6)$ \\
& $p$-value & 0.099 & 0.062 & 0.077 \\
\hline
\end{tabular}

Mann-Whitney U-test.

Table (2): Showing Rome III criteria for both groups before and after treatment sessions.

\begin{tabular}{lllll}
\hline & & Before & After & After \\
treatment & sessions & 6 months \\
\hline $\begin{array}{c}\text { Two or fewer defecations in the } \\
\text { toilet per week }\end{array}$ & Case group & 40 & 2 & 3 \\
& Control group & 40 & 13 & 14 \\
& $p$-value & 1 & $0.01^{*}$ & $0.01^{*}$ \\
At least one episode of fecal & Case group & 12 & 3 & 3 \\
incontinence per week & Control group & 10 & 3 & 4 \\
& $p$-value & 0.189 & 1 & 0.987 \\
History of painful or hard & Case group & $30 / 40$ & $5 / 40$ & $6 / 40$ \\
bowel movements & Control group & $28 / 40$ & $12 / 40$ & $15 / 40$ \\
& $p$-value & 0.177 & $0.033^{*}$ & $0.028^{*}$ \\
History of retentive posturing & Case group & $10 / 40$ & $3 / 40$ & $2 / 40$ \\
or excessive volitional stool & Control group & $12 / 40$ & $8 / 40$ & $6 / 40$ \\
retention & $p$-value & 0.189 & $0.049 *$ & $0.048^{*}$ \\
Presence of a large fecal mass & Case group & $11 / 40$ & $2 / 40$ & $3 / 40$ \\
in the rectum & Control group & $12 / 40$ & $10 / 40$ & $12 / 40$ \\
& $p$-value & 0.199 & $0.025^{*}$ & $0.022^{*}$ \\
\hline
\end{tabular}

\section{Discussion}

Chronic functional constipation remains a troublesome condition to treat and children refractory to conventional treatments confront surgical procedures [11]. Our patients meet Rome III criteria for functional constipation [7]. Standard treatment with laxatives and enemas was not effective. However, their symptoms significantly improved with the IF current therapy, with no adverse effects of treatment were reported.

It was hypothesized that an interferential current may influence the neuroplasticity of the intestinal nerves, inducing structural, intrinsic or synaptic changes leading to altered neuronal function; others proposed that IF current causes improved propagating sequences and better colonic activity [12], but prove has yet to be found to decide the exact mechanism of action. One speculation is that interferential stimulation causing its effects through electrically stimulating excitable cells, which deliver slow wave activity in the bowel accountable for intestinal movements, or that it directly stimulates the nervous system [13]. Low-frequency currents, which are needed to stimulated nerves, result in high skin resistance. Skin impedance is inversely proportional to the frequency of the current. To overcome this, a higher current is needed, which can cause pain. In distinction, a high-frequency 
current is associated with low skin resistance and passes through without pain [14].

The present study demonstrated that addition of IF electrical current stimulation to the conventional rehabilitation program improves the effectiveness of the treatment among participants, Table (1). Both groups have shown progress, however, the IF therapy group showed a greater proportion of increased frequency of defecation, reduction of soiling and pain this advantage lasted throughout the followup period. Adding IF electrical stimulation to PFM exercises resulted in significant increase in the frequency of weekly bowel movement in the case group from 3 days/week to 7 days/week at end of sessions in relation to the controls which showed no improvement. Our finding is in agreement with other studies $[\mathbf{1 5 , 1 6}]$, that had used IF current to treat constipation not only in children but also in adults.

Out of sixty-two cases of pediatric resistant slow transient constipation, using home device for IF current stimulation Yik et al., [17] showed improvement in defecation frequency in $91 \%$, soiling frequency decreased from 4.8 to 1.1 days/week, abdominal pain decreased from 1.7 to 0.3 days /week, urge to defecate improved and quality of life in half of their chronic patients. Our results showed that fecal soiling/day was reduced in both groups but significantly more in the case group than the control group.

In a previous study, constipation symptoms were improved in children with treatment-resistant constipation and isolated holdup in the anorectum by transcutaneous electrical stimulation [18]. It was postulated that transcutaneous electrical stimulation produced a global clinical improvement of bowel function in a group of children with chronic constipation, who had anorectal retention, with increased defecation frequency to a normal range in $50 \%$ of children, reduced fecal incontinence episodes, and improvement in their QOL in $90 \%$ of them [18]. In the present study, quality of life showed improvement in both groups but we could not detect more improvement after the end of the treatment sessions or at end of the follow-up period.

In children post-surgery for Hirschsprung disease, IF treatment plus behavioral therapy was more successful in that it normalizes stool form, reducing frequencies of fecal incontinence and improving the frequency of defecation than behavioral therapy alone [4].

Studying the effect of physiotherapy added to medical treatment vs. medical treatment alone was done by Silva and Motta [19] in 72 children, their age range between 4 and 18 years old, suffering from functional constipation diagnosed by Rome III criteria 6 weeks after treatment, there were significantly higher defecation frequency in the group who physiotherapy was added to medical treatment (5.1 days/week) compared with the medical treatment group (3.9 days/week) $(p=0.01)$, although fecal incontinence frequency remained unaffected in these patients [19]

Sharifi-Rad et al., [4] treatment success was achieved for $88.4 \%$ of children in the case group they studied compared with $43.2 \%$ of children in the control group after the treatment $(p<0.003)$. The constipation score was reduced in both groups they studied. Stool form was normalized in $75.6 \%$ of their cases and $45.5 \%$ of the controls after the treatment.

We thought that PFM exercise is considered a simple method to increase children awareness about their pelvic floor muscles and teach children to relax their muscles during defecation. It enabled the successful introduction of IF stimulatory current, although it may be insufficient especially in FC resistant cases.

In conclusion, IF stimulatory current was a non-invasive effective treatment and was associated with a better increase in the efficiency of treatment of chronic functional constipation. Defecation frequency was increased in our patients and was associated with decreased soiling, reduced pain and improved quality of life. It should be added as part of the rehabilitation program of the resistant cases of FC.

\section{Recommendations:}

In the future, use of portable IF devices may prevent more invasive measures such as surgery for children suffering from functional constipation.

\section{References}

1- MUGIE S.M., BENNINGA M.A. and DI LORENZO C. Epidemiology of constipation in children and adults: A systematic review. Best Pract. Res. Clin. Gastroenterology, 25: 3-18, 2011.

2- DEHGHANI S.M., KULOUEE N., HONAR N., IMANIEH M-H., HAGHIGHAT M. and JAVAHERIZADEH H.: Clinical manifestations among children with chronic functional constipation. Middle East J. Dig. Dis., 7: 31$5,2015$.

3- VOSKUIJL W.P., VAN DER ZAAG-LOONEN H.J., KETEL I.J., GROOTENHUIS M.A., DERKX B.H. and BENNINGA M.A.: Health related quality of life in disorders of defecation: The Defecation Disorder List. Arch. Dis Child., 89: 1124-78, 2004. 
4- L. SHARIFI-RAD, S.S. LADI-SEYEDIAN, N. MANOUCHEHRI, H. ALIMADADI, B. ALLAHVERDI, F. MOTAMED and G-H. FALLAHI: Effects of Interferential Electrical Stimulation plus Pelvic Floor Muscles Exercises on Functional Constipation in Children: A Randomized Clinical Trial. The American journal of gastroenterology- nature.com. Dec., 19, 2017.

5- JUDITH S. MOORE, PETER R. GIBSON and REBECCA E. BURGELL: Neuromodulation via Interferential Electrical Stimulation as a novel Therapy in Gastrointestinal Motility Disorders J. Neuro-gastroenterol. Motil. Jan., 24 (1): 19-29, 2018.

6- VAN WUNNIK B.P., PEETERS B., GOVAERT B., NIEMAN F.H. and BENNINGA M.A., et al.: Sacral neuromodulation therapy. A promising treatment for adolescents with refractory functional constipation. Dis. Colon. Rectum., 55 (3): 278-285, 2012.

7- BOCCIA G., MANGUSO F., COCCORULLO P., MASI P., PENSABENE L. and STAIANO A.: Functional defecation disorders in children: PACCT criteria versus Rome II criteria. J. Pediatric., 151 (4): 394-398, 2007.

8- FICHTNER-FEIGL S., SAILER M., HÖCHT B., et al.: Development of a new scoring system for the evaluation of incontinence and constipation in children Coloproctology, 25: 10-15, 2003.

9- CLARKE M.C1, CHOW C.S., CHASE J.W., GIBB S., HUTSON J.M. and SOUTHWELL B.R.: Quality of life in children with slow transit constipation. J. Pediatric. Surg. Feb., 43 (2): 320-4, 2008

10- CHASE J., ROBERTSON V.J., SOUTHWELL B., et al.: Pilot study using transcutaneous electrical stimulation (interferential current) to treat chronic treatment-resistant constipation and soiling in children. J. Gastroenterology Hepatol., 20: 1054-1061, 2005.

11- VAN DER WILT A.A., VAN WUNNIK B.P., STURKENBOOM R., et al.: Sacral neuromodulation in children and adolescents with chronic constipation refractory to conservative treatment. Int. J. Colorectal Dis., 31: 1459$1466,2016$.

12- CLARKE M.C., CHASE J.W., GIBB S., et al.: Decreased colonic transit time after transcutaneous interferential electrical stimulation in children with slow transit constipation. J. Pediatric. Surg., 44: 408-412, 2009.

13- SOUTHWELL B.R., YIK Y.I., TAN A., JORDAN-ELY J. and HUTSON J.M.: Transcutaneous electrical stimulation over the belly in slow-transit constipation. In: Nunez R, Fabbro MA, editors. Constipation in children: Diagnosis and treatment. Hauppause: Nova Science Publishers Inc., 2013.

14- OZCAN J., WARD A.R. and ROBERTSON V.J.: A comparison of true and premodulated interferential currents. Arch. Phys. Med. Rehabil., 85: 409-415, 2004.

15- QUERALTO M., VITTON V., BOUVIER M., et al. Interferential therapy: A new treatment for slow transit constipation. A pilot study in adults. Colorectal Dis., 15: e35-e39, 2013.

16- VITTON V., BENEZECH A., HONORÉ S., et al.: CONCOUR study: Interferential therapy in the treatment of chronic constipation in adults: Study protocol for a randomized controlled trial. Trials, 16: 234, 2015.

17- YIK Y.I., HUTSON J. and SOUTHWELL B.: HomeBased Trans abdominal Interferential Electrical Stimulation for Six Months Improves Pediatric Slow Transit Constipation (STC). Neuromodulation. Nov., 22, 2017.

18- YIK Y.I., STATHOPOULOS L., HUTSON J.M., et al.: Home Transcutaneous Electrical Stimulation Therapy to Treat Children with Anorectal Retention: A Pilot Study. Neuromodulation, 19: 515 -521, 2016.

19- SILVA C.A. and MOTTA M.E.: The use of abdominal muscle training, breathing exercises and abdominal massage to treat pediatric chronic functional constipation. Colorectal Dis., 15: 250-255, 2013.

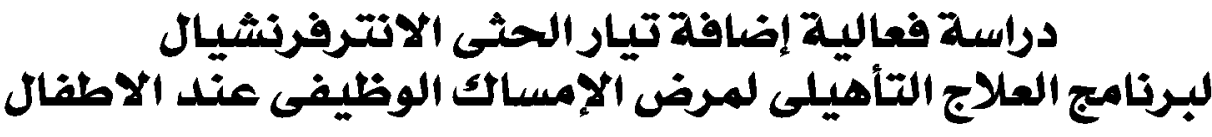

الأسـاليب: .ـ طفلاً يعانون من إمساك وظيفى مزمن وقد تم تاكيد التشخيص على أساس معايير روما الثالث. مجموعة العلاج، (العدد =

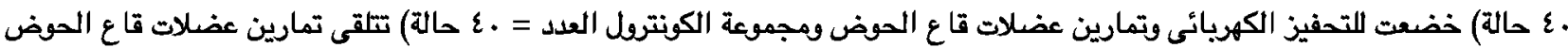

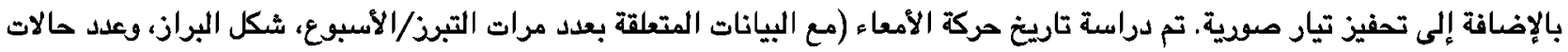

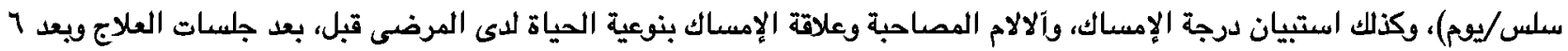
أشهر لجميع المشاركين.

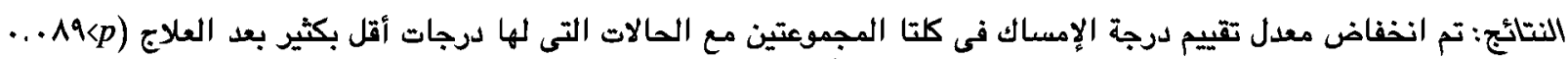

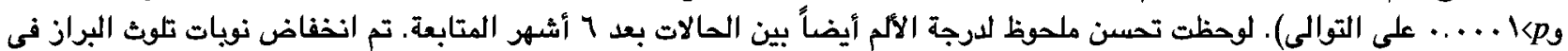

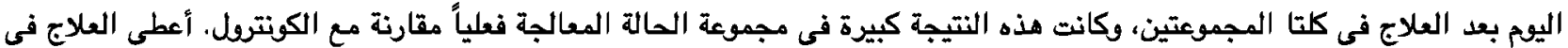

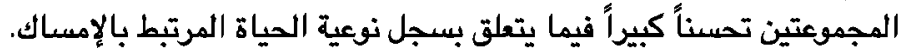

الخلاصة : ارتبط التيار التحفيزى إنترفرنشيال بتتائج أفضل فى فعالية علاج الحالات المزمنة التى تعانى من إمساك وظيفى مزمن وينبغى

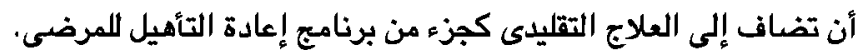

